AAS WORKING PAPERS IN SOCIAL ANTHROPOLOGY

Volume 5

Martin Gaenszle

Ritual und Verschriftlichung:

Ethnisierungprozesse in Ostnepal

Band 5

ÖAW ARBEITSPAPIERE ZUR SOZIALANTHROPOLOGIE 


\section{AAS Working Papers in Social Anthropology /}

ÖAW Arbeitspapiere zur Sozialanthropologie

ISSN: 1998-507X

doi: 10.1553/wpsa5

ISBN: 978-3-7001-6644-3

Wien 2009

\section{Editors / Herausgeber:}

Andre Gingrich \& Guntram Hazod

(C) Forschungsstelle Sozialanthropologie

Zentrum Asienwissenschaften und Sozialanthropologie

Österreichische Akademie der Wissenschaften

Prinz-Eugen-Straße 8-10

A-1040 Wien

Fax: 01/ 51581-6450

E-Mail: sozialanthropologie@oeaw.ac.at 


\title{
RITUAL UND VERSCHRIFTLICHUNG:
}

\author{
Ethnisierungprozesse in Ostnepal
}

\author{
Martin Gaenszle \\ Universität Wien
}

Die Sprachen der Kiranti-Gruppen in Ostnepal gelten zum großen Teil als stark vom Aussterben bedroht. ${ }^{1}$ Dies liegt zum einen daran, dass es sich um relative „kleine“ Sprachen handelt: die über 30 Kiranti-Sprachen (die meisten davon Rai, dazu Limbu und Sunuwar) haben oft nur wenige Tausend Sprecher. Zum anderen ist die akute Bedrohung in der zunehmenden Dominanz der Nationalsprache Nepali begründet, die lange Zeit in den öffentlichen Medien und Schulen keine Minderheitssprachen neben sich duldete. Zwar ist das Phänomen des Sprachentodes komplex, und es wurde oft darauf hingewiesen, dass viele unterschiedliche Faktoren den Grad der Bedrohung ausmachen (s. Crystal 2000). Doch was immer die Zukunft bringen mag, es ist heute deutlich, dass die Angehörigen dieser Gruppen selbst die Gefährdung ihrer Sprachkultur immer bewusster wahrnehmen und dagegen kämpfen. Dabei spielt die orale Tradition eine wichtige Rolle: sie ist der Inbegriff einer eigenen Sprachtradition und eng verknüpft mit der religiösen Praxis. Das Bewusstsein der ethnischen Zugehörigkeit kommt darin in seiner Vielschichtigkeit am deutlichsten zum Ausdruck (s. Gaenszle 2000, 2002a). ${ }^{2}$

Angesichts der weltweit zunehmenden Problematik des Sprachentodes sind seit den 1990er Jahren eine Reihe von wissenschaftlichen Programmen zur Dokumentation solcher Sprachen eingerichtet worden. Die meisten davon konzentrieren sich auf die linguistische Beschreibung, d.h. Grammatik und Lexikon. Da sich jedoch immer wieder gezeigt hat, dass die umfassende Dokumentation einer Sprache auch die Vielfalt der oralen Genres berücksichtigen muss, haben einige Programme auch eine ethnologische Komponente. Ein solches Programm ist das von der Volkswagenstiftung geförderte DOBES („,Dokumentation Bedrohter Sprachen“). Dieses wurde im Jahr 2000 begründet und umfasst inzwischen (2008) mehr als vierzig Projekte weltweit. Dabei ist es ein explizites Ziel, dass die linguistische und ethnographische Dokumentation nicht nur wissenschaftlichen Zwecken dient, sondern auch die Möglichkeit gibt, den Sprachgemeinschaften zu helfen, ihre Sprachen zu erhalten und gegebenenfalls zu revitalisieren (z.B. durch Erstellung von Lehrmaterial). Das Ziel aller DOBES-Projekte ist die Erstellung eines digitalen Archives, welches am Max-Planck-Institut für Psycholinguistik in Nijmegen angesiedelt ist. Dort werden alle Aufzeichnungen (Video, Audio, Foto etc.) gespeichert und können nach Abschluss der Arbeiten für wissenschaftliche Zwecke zugänglich gemacht werden. Vor allem aber soll das Archiv für die Mitglieder der betroffenen Gemeinschaften nutzbar sein.

Eines der im Rahmen von DOBES geförderten Projekte ist das „Chintang and Puma Documentation Project" (CPDP), an dem ich seit dem Beginn 2004 als Verantwortlicher für den ethnologischen Teil mitarbeite (s. Gaenszle et al. 2005). Das Projekt basiert auf einer Kooperation der Universität Leipzig

\footnotetext{
${ }^{1}$ Der vorliegende Text wurde am 25.11.2008 an der ÖAW präsentiert. Es handelt sich dabei um eine teilweise abgewandelte Version eines Aufsatzes, der demnächst in englischer Sprache erscheinen wird: „Scripturalisation of Ritual'“, in: Brosius, Christiane und Karin Polit (Hg.), Ritual, Heritage and Identity: The Politics of Culture and Performance in a Globalised World. New Delhi: Routledge South Asia. Ich danke den Herausgeberinnen für ihr Einverständnis mit dieser online-Publikation.

${ }^{2}$ Siehe auch Hardman 2000, eine Monographie über die Lohorung Rai, die sich insbesondere mit dem Begriff der Person befasst.
} 
und der Tribhuvan Universität in Nepal und ist interdisziplinär angelegt: es umfasst Linguistik, Ethnologie und Spracherwerbsforschung.

Gegenstand der Dokumentation sind zwei Rai-Sprachen, die jeweils ca. 4000-5000 Sprecher umfassen. Chintang wird im Dhankuta Distrikt (Koshi Zone) in einem einzigen Dorfdistrikt gesprochen, Puma etwas weiter westlich, im Khotang Distrikt. Die Rai-Gruppen dieser Region (etwas südöstlich des Mt. Everest-Gebietes gelegen) gehören zur indigenen Bevölkerung Ostnepals, die sich heute auch als Adivasi bezeichnet, und die erst im Zuge der Shah-Invasion Ende des 18. Jahrhunderts unter die Herrschaft der Könige Kathmandus gelangte. Der Einfluss der Nepali-Sprache ist also noch relativ jung, gleichwohl gab es jedoch verschiedene linguistische Berührungen mit Sprachen, die weiter südlich gesprochen werden (insbesondere Maithili).

Die Kiranti-Sprachen wurden schon im 19. Jahrhundert von Brian Hodgson, dem vielseitig interessierten britischen Residenten in Kathmandu entdeckt und als besondere Sprachfamilie identifiziert(s. Waterhouse 2004). Eine linguistische Besonderheit dieser Sprachen ist eine stark ausgeprägte Verbalkongruenz, was früher auch als „komplex pronominalisiert“ umschrieben wurde: dies heißt, dass Endungen transitiver Verben sowohl mit dem Agens als auch mit dem Patiens kongruieren.

Vor allem aber, und dies ist für Ethnologen von besonderem Interesse, besitzen diese Gruppen eine ausgeprägte ritualsprachliche Tradition. Für die Anrufungen der Ahnen und Ahnengottheiten wird ein bestimmtes Register verwendet, das sich von der Alltagssprache unterscheidet. Da die linguistische Kompetenz der Verwendung dieser Sprache auf wenige Experten beschränkt ist, ist diese Tradition in besonderem Maße bedroht. Gleichzeitig ist die Erhaltung dieser Sprachform für die Gruppenmitglieder von besonderem Interesse, da sie die eigene Religion verkörpert und die Differenz zu Hochreligionen wie Hinduismus und Buddhismus zum Ausdruck bringt.

Hier wird nun deutlich, dass ein solches Projekt der Sprachdokumentation nicht in einem politischen Vakuum steht. Vielmehr ist die orale Tradition ein wichtiges Medium der ethnischen Selbstdarstellung und Selbstbehauptung, mithin der Ethnisierung, und wie weiter unten gezeigt werden soll, hat diese eine Vorgeschichte, die weit zurückgeht. Die orale Tradition der Kiranti kann als paradigmatischer Fall eines „immateriellen Kulturerbes“ (intangible heritage) betrachtet werden, das konstitutiv für das ethnische Selbstverständnis ist. Aus diesem Grund steht die Tradition heute im gegenwärtigen Nepal im Blickpunkt der öffentlichen Selbstdarstellung dieser Gruppen. Wie ich im Folgenden zeigen möchte, ist die orale Tradition aufgrund des politischen Kontextes vielfachem Druck ausgesetzt, vor allem aufgrund der Versuche, das Medium des Schreibens in der Form einer eigenen Schrift einzuführen. ${ }^{3}$ Diese Einflüsse haben, auch wenn sie bislang marginal waren und relativ wenig Einfluss auf die rituelle Praxis außerhalb der Städte hatte, eine neue Bewertung der religiösen Tradition zur Folge gehabt. Und dies ist gerade im kompetitiven Kontext der heutigen ethnischen Politik von weit reichender Bedeutung, zumal die Kiranti sich als eine von vielen Minderheiten in einer demokratisierten nationalen Arena zu behaupten haben.

Die orale Tradition der Kiranti ist bekannt unter dem Begriff mundum oder muddum, bzw. einiger anderer verwandter Termini, die in den verschiedenen Untergruppen differieren (s. ridum, mindum, pelam). ${ }^{4}$ Aber die Kernbedeutung ist in allen gleich: die Tradition ist nicht nur eine Überlieferung von

\footnotetext{
${ }^{3}$ Mein Ansatz kann als Teil einer „Ethnographie des Schreibens“ betrachtet werden, wie sie Keith Basso in seinem frühen programmatischen Aufsatz skizziert hat (Basso 1974).

${ }^{4}$ Dieser Begriff wird in meiner Monographie über die Mewahang Ritualtexttradition näher analysiert (Gaenszle 2002a: 31-56).
} 
Texten, sondern die eines Wissens und einer Lebensform, die von den Ahnen etabliert wurde und als heilig und bindend betrachtet wird - als eine Art kultureller Ethos. Die verschiedenen Varianten des Begriffs verweisen auf eine gemeinsame Wurzel *dum, die möglicherweise mit Tib. sgrung verwandt ist (,Legende', ,Fabel', von einem Barden gesungene Geschichte'). Eine Variante, pelam, ist vermutlich mit Tib. dpe verwandt (,Muster', ,Modell', ,Parabel'), - ähnliche Begriffe gibt es bei den Gurung (pe-da lu-da) und Tamang (pe:thim).

Der entscheidende Punkt ist, dass alle diese Traditionen im Wesentlichen oraler Natur sind. Sie wurden in mündlicher Weise überliefert, und es ist erst seit relativ kurzer Zeit, dass man damit begonnen hat, die Tradition zu verschriftlichen. Dieser Prozess der Inskription kann auch als Vertextlichung umschrieben werden. Sheldon Pollock prägte dafür den Begriff der „literization“(Verschriftlichung), und unterscheidet diesen von „literarization“, d.h. der Verwandlung in Literatur (Literarisierung). Dieser Prozess war im Indien des „,vernacular millenium“ verbunden mit einer Homogenisierung und Ethnisierung (Pollock 1998: 41). Hier in unserem Fall von Ostnepal ist damit noch etwas Weiteres verbunden, nämlich die Schaffung eines schriftlichen Kanons (scripture). Dies impliziert einen religiösen Gebrauch der schriftlichen Texte, oft im Rahmen von Ritualen. Dieser Prozess hat hier erst begonnen, aber eine gewisse Tendenz ist schon abzusehen. Um diesen Prozess analysieren zu können, werde ich zunächst den allgemeinen politischen Hintergrund betrachten (1.). Dann werde ich die Erfindung der Kiranti Schrift näher betrachten (2.), sowie ihre darauf folgende Verbreitung in der Kiranti Sprachbewegung (3.). Schließlich wende ich mich noch der Bedeutung der Schrift im gegenwärtigen Kontext der ethnischen Politik zu (4. und 5.).

\section{Der politische Kontext der Identitätsbildung in Ostnepal}

In jüngerer Zeit, vor allem seit den frühen 1990er Jahren, haben die Kiranti Ostnepals eine ethnische Identität auf institutioneller Basis geschaffen. Sie haben ethnische Organisationen gegründet, wie „Kirat Rai Yayokkha“ (Rai), oder „Yakthung Chumlung“(Limbu), welche die Interessen der „tribalen“ ethnischen Gruppen in einem nationalen Kontext vertreten. Die Rai Organisation Kirat Rai Yayokkha wurde 1990 gegründet und ist Teil der „Nepal Federation of Indigenous Nationalities“ (NEFIN), der Dachorganistion aller ethnischen Minoritäten (vormals Nepal Janajati Mahasangh). Die Organisation der Rai ist landesweit gut organisiert und hat Niederlassungen in den meisten Distrikten des Ostens. Das Zentralbüro ist in Kathmandu, von wo aus die Arbeit koordiniert wird.

Ein zentrales Problem dieser ethnischen Organisation ist, dass die Rai eine große Zahl von distinkten Sprachen sprechen. Es gibt mehr als 20 Untergruppen, die alle eine eigene (also wechselseitig nicht verständliche) Sprache sprechen. Deshalb war und ist es schwierig, eine solidarische Einheit zu schaffen. Gegenwärtig ist Yayokkha von einer bestimmten Rai-Gruppe dominiert, nämlich den Bantawa Rai. Diese sind zahlenmäßig eine der größten Rai-Gruppen und besetzen heute die wichtigsten Positionen in der Organisation - was schon gewisse Spannungen mit sich brachte.

Die Rai in dieser Organisation haben ein neues Selbstbild erschaffen: es gibt eine große Zahl von lokalen Publikationen, die die Geschichte und Kultur der eigenen Gruppe beschreiben und ihre Herkunft rühmen. So wird z.B. mit Stolz darauf verwiesen, dass die Kiranti (also auch die Rai) die direkten Nachfahren der Kirã̃ta Dynastie seien, die einstmals (vor unserer Zeitrechnung) das Kathmandu-Tal beherrschten. ${ }^{5}$ Die Kultur wird in den Pamphleten als egalitär und hoch zivilisiert beschrieben: es gab

\footnotetext{
${ }^{5}$ Zum historischen Hintergrund der Entwicklung des Exonyms „Kiranti“" s. Gaenszle 2000: 4-8.
} 
demnach keinerlei Hierarchien, wie etwa im Kastensystem der Hindus (vgl. Schlemmer 2004). Diese Bücher betonen also, dass die Kiranti vor langer Zeit eine ausgeprägte Zivilisation besaßen, die dann allerdings verloren ging, als sie aus dem Kathmandu-Tal vertrieben wurden (Licchavi).

Diese hoch entwickelte Zivilisation, so heißt es, habe auch eine reiche Schriftkultur besessen, und einen Kanon religiöser Texte. Diese sind auch als „Kirant ko veda“ bekannt: die Veden der Kiranti ein Text, der von einem Limbu in den 1940er Jahren publiziert wurde (s.u.). Die Schrift, die damals genutzt wurde, sei jedoch aufgrund unglücklicher Umstände verloren gegangen und erst, wie ich noch ausführen werde, später „wiederentdeckt worden“.

Generell kann man diese neuen Publikationen als den Ausdruck eines neuen ethnischen Selbstbewusstseins sehen, das mit einer bestimmten politischen Agenda verbunden ist. Die Kiranti, die im späten 18. Jahrhundert von der Shah-Armee besiegt und dann lange unterdrückt wurden, wollen endlich wieder etwas zu sagen haben. Sie fordern teilweise Autonomie, zumindest aber eine angemessene Repräsentation im neuen föderalen Nationalstaat, der nun begonnen hat, eine neue Verfassung auszuarbeiten.

In dieser neuen öffentlichen Selbstdarstellung beziehen sich die Rai stark auf ihre orale Tradition. Die Rai verweisen hier meist auf den Mythos von Sumnima und Paruhang, die Geschichte des Urelternpaares, von dem die Schöpfung ausging und das als eine Art Rollenmodell die Welt der Ahnen repräsentiert. Paruhang, der im Mythos als Jäger beschrieben wird, wird heute oft als Kirāteśvar bezeichnet und somit als Form von Shiva gesehen, während Sumnima entsprechend als eine Form von Parvati dargestellt wird. Diese Identifikation schafft also eine Verbindung zur „Großen Tradition“ des Hinduismus.

Viele populäre Abbildungen stellen die Kiranti als ehemalige Herrscher des Kathmandu-Tals dar. So z.B. das Bild von König Yalambar, jenem Kirata, auf den die Gründung der Stadt Patan zurückgeführt wird. Typischerweise wird Yalambar als ein streitbarer Krieger dargestellt, bewaffnet mit Pfeil und Bogen. In einer Abbildung sieht er aus wie eine Mischung aus einem tibetischen gyelpo und einem Hindu rāja $\overline{\text {. }}$

Doch die ethnischen Organisationen veröffentlichen heute nicht nur Erzählungen über die frühe und jüngere politische Geschichte, sie bringen auch Bücher über die wichtigsten Rituale heraus: Beschreibungen der rituellen Abläufe, wie auch Texte der rituellen Anrufungen. Hier muss angemerkt werden, dass die Kiranti im Ritual eine distinkte Ritualsprache verwenden, die sich von der Alltagssprache abhebt. Diese Sprache ist reich an Parallelismen (syntaktisch, phonologisch, morphologisch) und besitzt zum Beispiel eine bestimmte Nominalform, die Binomiale, die nur hier Verwendung finden (Gaenszle 2000a). Dieser sprachliche Code gilt als einzig angemessen bei der Kommunikation mit den Ahnen und kann als eine Form der Höflichkeit und Respekterweisung gesehen werden. Die rituellen Anrufungen des muddum sind bis heute das Kernstück der religiösen Praxis in den Dörfern. Ohne die richtige und regelmäßige Beopferung der Ahnen und Ahnengottheiten mithilfe dieser sprachlichen Darbietung würde in der Sicht der Kiranti Unheil drohen, denn dies würde den Zorn der Vorfahren zur Folge haben.

Doch um zu verstehen, wie diese rituelle Sprachform in eine Schrifttradition verwandelt und dadurch ein neues prestigehaltiges Erbe geschaffen wird, müssen wir uns zunächst mit der Einführung oder Erfindung der Schrift befassen, und mit ihrem Gebrauch im Laufe der Sprachbewegung der Kiranti. 


\section{Srijanga und die Erfindung der Kiranti-Schrift}

Die historischen Anfänge dieser Schrift sind im 18. Jahrhundert zu finden, als ein Limbu Mönch mit dem Namen Srijanga (Śrijanga, auch Sirihariga und ähnliche Schreibweisen) eine eigene Schrift zu verbreiten begann. ${ }^{6}$ Für die Kiranti ist Srijanga der „Wiederentdecker“ der alten verloren gegangen Schrift ihrer Vorfahren. Denn einer beliebten Erzählungen zufolge gab es diese Schrift schon bis ins späte erste Jahrtausend unserer Zeitrechnung, ist dann aber über 800 Jahre ,verloren“ gegangen, bis sie schließlich wieder entdeckt wurde (s. auch Subba 1995).

Doch für diese Geschichte gibt es keine historischen Belege. Vielmehr weist alles darauf hin, dass die Schrift von jenem Srijanga selbst erfunden wurde. In den Hodgson-Dokumenten (gesammelt vom oben genannten britischen Residenten Brian Houghton Hodgson in den 1840er Jahren) finden sich Informationen zu diesen Ereignissen, die nahe legen, dass Srijanga, der auch als Rupihang bekannt war, von den Machthabern in Sikkim als Separatist gesehen wurde (Dhungel 2006). Offenbar war er ein monastisch gebildeter Mann, der seinen Limbu-Brüdern ein Medium zur Verfügung stellen wollte, mit dem sie ihre Tradition niederschreiben konnten, um diese als etwas Eigenständiges zu präsentieren. Doch, wie es aussieht, sahen die Herrscher von Sikkim dies nicht gerne, zumal sie die hoheitliche Kontrolle über das Limbugebiet in Ostnepal beanspruchten. Srijanga wurde schließlich ermordet, und alles deutet darauf hin, dass dies politische Hintergründe hatte.

Diese Geschehnisse zeigen, dass der Besitz einer Schrift nicht allein eine religiöse Angelegenheit, sondern auch in hohem Maße mit sozialem Prestige verbunden war und somit ein sehr begehrtes Gut war. Ähnliche Geschichten der ,verlorenen Schrift“ gibt es im Übrigen in vielen Teilen des Himalaya, auch Tibet und sogar in Burma und Kambodscha, wie Michael Oppitz (2006) gezeigt hat. Immer wird dabei eine Begründung gegeben, weshalb die Schrift durch unglückliche Umstände verloren gegangen ist: manchmal wurden die Texte verbrannt, mal aus Versehen gegessen, oder einfach verspielt. Wie auch immer, die Mythen bringen ein verbreitetes Gefühl zum Ausdruck, dass orale Kulturetwas Minderwertiges sei, für das man sich schämen muss, dass aber Schrift ursprünglich existiert hatte und somit die Tradition „eigentlich“ keine orale war. Offenbar entstand eine solche Einschätzung in Situationen, da mündliche Überlieferungen auf Schrifttraditionen trafen, gegenüber denen sie sich rechtfertigen mussten - wie beim Kontakt mit hinduistischen, buddhistischen oder christlichen Traditionen.

Heute wird Srijanga in Ostnepal als ein Held, als eine Art Kulturheros gefeiert, der oft in der SanktSebastian-Pose dargestellt wird, durchbohrt von Pfeilen, fast wie ein christlicher Märtyrer. So steht am Eingang der Distrikthauptstadt von Sunsari, in Dharan, wo ein großer Bevölkerungsanteil zur wohlhabenden Kiranti Mittelschicht gehört, seit kurzem eine große Statue von Srijanga. Dieses Denkmal hat eine klare Botschaft: unser Vorfahre wurde von den Herrschenden getötet, aber seine Leistung als Kulturheros hat überlebt.

Die von Srijanga entwickelte Schrift ist nur in wenigen Dokumenten erhalten. Soweit wir wissen, wurde sie im 19. Jahrhundert wenig genutzt, zumindest nicht als Medium der Überlieferung. Doch schließlich zu Beginn des 20. Jahrhunderts gab es ein regelrechtes Revival, eine weitere „Neuentdeckung“ dieser Schrift. Vor allem war es Iman Singh Chemjong, der die Schrift revidierte und einige Buchstaben hinzufügte. Er ging in Darjeeling und auch Ostnepal umher, um die Schrift zu verbreiten. Chemjong war in einer Mission School in Calcutta ausgebildet worden, und so war

${ }^{6}$ Eine ausführlichere Untersuchung der Kiranti-Sprachbewegung und inbesondere der Rolle Phalgunandas (s.u.) findet sich in Gaenszle 2007. 
er christlichen Einflüssen ausgesetzt. Aber er war zusammen mit Angehörigen der Limbu-Eliten in Darjeeling ein begeisterter Anhänger der Kiranti-Bewegung, die für eine kulturelle Erneuerung kämpfte. ${ }^{7}$ In dieser Zeit (ca. 1920-1940) war das koloniale Darjeeling ein Zentrum des ethnischen Aktivismus, wo z.B. zahlreiche Schulen eröffnet wurden, in denen eine neue Form der Bildung angeboten wurde. So wurde z.B. die älteste Limbu Organisation, Yakthunghang Chumlung Sabha, 1925 gegründet. Von ihr wurde die „Jambok Memorial School“ betrieben (Subba 1995: 33). In diesem Zusammenhang spielte die Neuentdeckung der Kirantischrift eine wichtige Rolle. Zahlreiche Bücher wurden in der reformierten Schrift publiziert. Die Saat des ethnischen Aktivismus keimte also schon lange vor der Unabhängigkeit Indiens.

\section{Phalgunanda und die Satya Hangma Bewegung}

Dies zeigt also, dass es zu jener Zeit rege Aktivitäten gab, vor allem was die Produktion gedruckter Texte betraf. Die Veröffentlichung von Texten in der Kiranti-Schrift wurde zu einem Geschäft - ganz in der Art von Benedict Andersons , print capitalism“ (1983). ${ }^{8}$ Eine Reihe von Texten wurde weiterhin mit der Hand kopiert. Diese sind heute meist in Privatsammlungen, doch einige davon sind in den nationalen Archiven in Kathmandu zu finden. Das Zentrum der Aktivitäten war zwar Darjeeling, doch ungefähr zur gleichen Zeit begann eine ähnliche Bewegung in Ostnepal. Phalgunanda Lingden, ein Limbu, der 1885 in Panchthar in einfachen Verhältnissen als Nardhoj Lingden geboren wurde, floh als junger Mann aus seinem Dorf und schloss sich eine Zeitlang der britischen Armee an, was ihn nach Burma und schließlich im Ersten Weltkrieg auch nach Europa brachte. Aber er zeigte schon früh spirituelle schamanische - Neigungen und hatte schließlich, der Legende nach, in Italien ein Schlüsselerlebnis, das ihm die Sinnlosigkeit des Krieges offenbarte: da er sah, dass die gegnerischen Soldaten Menschen wie er waren, schoss er in die Luft und in die Erde bis seine Munition zu Ende war - auf wundersame Weise blieb er dabei unverletzt. Er kehrte nach Nepal zurück, wurde zu einem Wanderasketen und pilgerte einige Jahre, meist zusammen mit einer wachsenden Gruppe von Anhängern, durch den Himalaya. Eines Tages machte er schließlich eine Entdeckung: er fand, so die Geschichte, Inschriften der Kiranti-Schrift auf einem Felsen. Daraufhin begann er, diese Schrift zu lernen und schließlich aktiv zu verbreiten. Dies war in einer Zeit, als einige Limbu-Oberhäupter in Ostnepal begonnen hatten, ihre „tribale“ Kultur zu reformieren, da sie die Notwendigkeit sahen, der dominanten Hindu-Tradition eine eigene Kiranti-Tradition entgegenzusetzen. Im Jahre 1931 fand ein großes Treffen statt, das "Yakthung Cumlung Satya Dharma Muculka", auf dem die Limbu-Eliten beschlossen, einige wesentliche Elemente ihrer Kultur zu verändern. Phalgunanda war einer der maßgebenden Anführer dieses Treffens und wurde der wichtigste Vertreter der neuen Ideen. Er wanderte umher und versuchte seine Kiranti-Brüder dazu zu bringen, dass sie aufhörten Fleisch zu essen und Alkohol zu trinken, und stattdessen einen „wahren“ und „reinen“ Lebensstil zu führen. Diese neue Religion wurde als satya dharma (,dharma der Wahrheit/ Wahrhaftigkeit') bezeichnet, die Gottheit im Zentrum war Satya Hangma (wörtlich ,Göttin der Wahrheit', wobei hangma auch die Bedeutung von ,Königin’ hat). Die „Entdeckung der Schrift“ gab ihm nun die Gelegenheit, sich nicht nur für das Unterrichten des Lesens und Schreibens, sondern

\footnotetext{
${ }^{7}$ Ich habe die historiographische Tätigkeit Chemjongs in Gaenszle 2002b näher beschrieben.

${ }^{8}$ Die Situation eines entstehenden Druckkapitalismus war hier ähnlich wie jene, die von Chalmers (2003) im Falle von Banaras im Zusammenhang mit der Entstehung der Nepali-Sprachbewegung beschrieben wurde. Doch während in Banaras die Nepali Publikationen eng mit dem entstehenden Nationalismus verbunden waren, beschränkten sich die Kiranti Veröffentlichungen auf ethnischen Aktivismus. Eine interessante Fallstudie der Auswirkungen der Druckrevolution in einer anderen südasiatischen Region und eine kritische Reflektion von Andersons wichtigem Buch finden sich in Blackburn 2001.
} 
generell für Schulbildung einzusetzen, die in der Zeit der Rana-Herrschaft der breiten Bevölkerung verwehrt war. Phalgunanda war also auch ein sozialer Reformer, und es überrascht deshalb nicht, dass er zunehmend von den Ranas als gefährlicher Widerständler wahrgenommen wurde. Er wurde sogar im Jahr 1938 als ,anti-nationaler“ (räjdrohi) Rädelsführer festgenommen und vor dem Distriktgericht von Dhankuta zur Rede gestellt. Man musste ihn jedoch mangels Beweisen laufen lassen.

Die Satya Hangma Bewegung, wie sie schließlich genannt wurde, war in den 1940er Jahren in Ostnepal sehr populär, nicht zuletzt da Phalgunanda seinen Kiranti-Brüdern in Aussicht stellte, dass sie, sobald sie ihren Lebensstil ändern würden, ihren kipat-Landbesitz (eine Form des kommunalen Bodenrechts), den sie zunehmend aufgrund von Verschuldung an hochkastige Hindus abtreten mussten, ${ }^{9}$ zurückerhalten würden. Dies war ein großer Anreiz, sich der Bewegung anzuschließen. Doch nach Phalgunandas Tod im Jahre 1949 in Panchthar verlor die Bewegung an Momentum.

Es wird also deutlich, dass diese neue Religion, Satya Dharma, oder „die Religion der Wahrhaftigkeit“, im Wesentlichen eine typische Reformreligion war, das Resultat einer nahezu paradigmatischen Sanskritisierung: zahlreiche Praktiken und Begriffe des hochkastigen Hinduismus wurden übernommen und in die eigene Tradition integriert. Gleichzeitig jedoch wurde die eigene Tradition der Kiranti aufgewertet und die eigenen Ahnen und Gottheiten, insbesondere die eigene Schrift in den Vordergrund gestellt. Wichtig ist also, dass die Anhänger Phalgunandas ihre Religion nicht als eine Form des Hinduismus betrachteten, obgleich der Mahaguru (,großer Lehrer', wie er auch tituliert wurde) mit einem triśul (Dreizack, Symbol Shivas) durch die Gegend zog und wie ein Śaiva Asket aussah. Er propagierte den Gebrauch der eigenen Schrift der Kiranti, sowohl in der Schulbildung als auch für die Rezitationen und Anrufungen der Ahnen. Die neue Religion war also eine synkretistische Mischung mit sowohl tribalen als auch hinduistischen Merkmalen. Dabei spielte die Kiranti-Schrift eine zentrale Rolle: sie war Inbegriff des Eigenen, und wurde fast als magisches Medium verehrt. Dies kann als eine Art Grapholatrie gesehen werden, wie sie auch aus dem tibetischen Kontext bekannt ist. ${ }^{10}$

Phalgunandas Satya Hangma Bewegung verschwand fast vollständig nach seinem Tod, auch weil in den 1950er Jahren die politische Situation sich grundlegend gewandelt hatte und nun der Aufbau eines demokratischen Nepals im Vordergrund stand. Doch die Bewegung wurde viele Jahre später revitalisiert, als Nachfolger des Mahaguru auftauchten. In den 1970er Jahren trat ein patrilateraler Verwandter (aus dem gleichen Clan der Lingden) in Erscheinung, mit dem Namen Atmananda, der behauptete, eine Reinkarnation von Phalgunanda zu sein. Dieser Atmananda scharte eine schnell wachsende Anhängerschaft um sich, betreibt heute ein gut florierendes Ashram in Larumba. Er ist zweifellos der bekannteste Anführer der Bewegung, doch interessanterweise nicht der einzige. Ein gewisser Jyotinanda, ein Rai aus Ilam, behauptete zwar nicht, eine Reinkarnation zu sein, doch trat er ebenfalls in die Fußstapfen von Phalgunanda und sah sich als Nachfolger. Er propagierte in den 1970er Jahren jedoch eine eigene Schrift der Rai, distinkt von der Kiranti-Schrift (die heute zunehmend als Limbu-Schrift bezeichnet wird). Und schließlich tauchte auch noch eine dritte Person auf, ebenfalls ein Rai, der trotz der anderen ethnischen Herkunft, den Anspruch erhebt, eine Reinkarnation von Phalgunanda zu sein. Sein Name ist Omnanda, ein relativ junger Mann, der 1979 in Mainamaina (Udaipur Distrikt) geboren wurde. Diese Entwicklungen zeigen, dass die Rai inzwischen einen eigenen Zweig der Satya-Hangma-Bewegung gebildet haben: d.h. die neue Religion hat sich aufgrund ethnischer Gesichtspunkte aufgespalten (Rai vs. Limbu). Omnanda ist heute der Repräsentant einer neuen Sekte, die für die Rai attraktiv ist.

\footnotetext{
${ }^{9}$ Siehe dazu die Fallstudie von Caplan 1970.

${ }^{10}$ Ein anderer Titel war tapasvin (,Asket').
} 


\section{Neue Anwendungen einer nicht so alten Schrift}

Es gibt heute also zwei Schriften, eine der Limbu (die von I.S. Chemjong weiterentwickelte KirantiSchrift von Srijanga), und eine der Rai. Letztere wurde erst in den 1960er Jahren in Sikkim von einem gewissen Kripashalyan Rai entwickelt und ist heute dort offiziell als Schrift der Rai anerkannt: seit 1999 werden in diesem indischen Bundesstaat Schulbücher in der Schrift gedruckt, und Schulkinder können sie lesen und schreiben lernen.

Es wird also deutlich, dass es sich hier zunehmend um eine Konkurrenz der Schriften handelt. Der „Sikkim Herald“ wird in nicht weniger als 11 Schriften gedruckt. Die Frage ist jedoch, wie diese Schriften tatsächlich verwendet werden. Um den schulischen Gebrauch der indigenen Schriften (eine wichtige Forderung der ethnischen Aktivisten) zu begründen, mussten nun auch Bücher produziert werden. Dies führte zu einer Welle von Veröffentlichungen in den ethnischen Schriften. Da jedoch viele Leser eher die am meisten verbreitete südasiatische Schrift Devanagari gewöhnt sind, entstanden auch viele bi-literale Publikationen. Eine von vielen kopierte Form der Darstellung ist die von Vairagi Kainla, einem Schriftsteller, der auch als ethnischer Publizist aktiv ist. Eines seiner zahlreichen Bücher ist "Samsoghā Mundhum:Pretātmāko Ākhyān ra Anuṣthān" (1996), eine typische Dokumentation eines Limbu- Ritualtextes in Kiranti-Schrift, Devanagari-Transliteration und Nepali-Übersetzung.

Interessanterweise ist die Art der Präsentation durch westliche Wissenschaftler beeinflusst. Wie mir Vairagi Kainla sagte, ist er durch András Höfers Publikationen wie „Tamang Ritual Texts I“ (1981) inspiriert worden. Indigene Veröffentlichungen wie die von Kainla sind heute sehr gefragt, insbesondere bei den Kiranti der Mittelschicht, und werden als Ausdruck einer modernen Religion betrachtet. Die Zahl dieser Publikationen wächst, und entsprechend findet eine gewisse Auswahl statt, so dass sich im Laufe der Zeit ein Kanon entwickelt und eine gewisse Homogenisierung der eigentlich stark lokal variablen Tradition stattfindet. Dieser Prozess ist erst am Anfang. Soweit mir bekannt ist, werden diese Texte bislang noch nicht im Rahmen von Ritualen praktisch genutzt, etwa als Grundlage von Rezitationen. Doch ist dies vermutlich nur eine Frage der Zeit, zumal die orale Tradition immer mehr bedroht und in vielen Orten schon am Verschwinden ist. Die gedruckten und verschriftlichten Versionen werden im Zuge dieser Entwicklung wichtiger werden, und es ist wahrscheinlich, dass sie zunehmend bei der Überlieferung als Gedächtnisstütze an Bedeutung gewinnen. Dies hätte einen fundamentalen Wandel des Rituals zur Folge, da dies die Form des Sprechens fixieren und die performativen Aktivitiäten wesentlich einschränken würde (s. Schiefflin 1998).

Die Tradition, welche ursprünglich vollständig mündlicher Natur war, wird nun also in jüngerer Zeit, zunehmend verschriftlicht und in einen schriftlichen Kanon verwandelt. ${ }^{11}$ Es entsteht eine Art von neuem sakralem Schrifttum. Eine eigene Schrift zu haben, wird als notwendiges Zeichen von „Zivilisation“ betrachtet. Zwar wurde die Kiranti-Schrift historisch betrachtet erst im 18. Jahrhundert erfunden, doch in der Sicht der Limbu existiert sie seit frühesten Zeiten. Gleichzeitig wird, wie wir sahen, die eigene Schrift mit Vorstellungen von Bildung und Reform verknüpft. Sie wird nicht nur für religiöse Zwecke genutzt, sondern auch für den schulischen Unterricht als Medium der Alphabetisierung. ${ }^{12}$ Damit wird das kulturelle Erbe mit Ideen von „Fortschritt“ in Verbindung gebracht, so dass die altehrwürdige Tradition gleichzeitig als „modern“ erscheint. Im gegenwärtigen

\footnotetext{
${ }^{11}$ Wie Robert Ekvall schon vor einiger Zeit betonte: „Among Tibetans, grapholatry is more real than idolatry” (Ekvall 1964: 114).

${ }^{12}$ Zum Prozess der Kanonisierung in Kontexten der frühen Geschichte, s. Assmann 1997: 103-129.
} 
Kontext der ethnischen Politik im Zuge der Schaffung einer neuen Verfassung für Nepal ist dies ein wichtiges symbolisches Kapital.

\section{Neue Literaturen}

Es muss jedoch betont werden, dass dies relativ neue Entwicklungen sind, und diese verlaufen bei den verschiedenen ethnischen Untergruppen der Kiranti nicht einheitlich. Vielmehr kann man sagen, dass sich am Verhältnis zur Schrift ethnische Differenzierungen ablesen lassen: die Schrift kann deshalb geradezu als Medium der Ethnisierung gesehen werden.

Während die auf Srijanga zurückgehende Kiranti-Schrift als ein Merkmal der Limbu-Kultur betrachtet wird, ist die Rai-Schrift außerhalb von Sikkim nur von wenigen Rai übernommen worden. In Nepal findet man stattdessen viele Publikationen, die für die Transkription von Rai-Texten Devanagari verwenden. Gerade die Zusammenarbeit mit Linguisten (wie auch im CPDP) hat inzwischen dazu beigetragen, dass viele lokale Sprachaktivisten eingesehen haben, dass eine eigene Schrift für die Verschriftlichung gar nicht nötig ist. Mit kleinen Modifikationen kann man jede der Rai-Sprachen problemlos darstellen (so können mit wenigen zusätzlichen Diakritika auch der glottal stop, oder unterschiedliche Vokale dargestellt werden). Die einzige Bedingung ist, dass man sich für ein System entscheiden muss und dieses dann konsistent einhält. Für viele Rai war diese Erkenntnis überraschend, da man immer davon ausgegangen war, dass man für jede Sprache eine Schrift braucht.

Dabei gibt es neue Formen der ethnischen Selbstdarstellungen, also der ethnischen Literatur. Ich möchte hier zum Schluss noch zwei Beispiele aus meinem eigenen Forschungsfeld betrachten, die zeigen sollen, in welche Richtung diese Entwicklungen verlaufen können (und auch: wie bei diesen Entwicklungen der Forscher involviert sein kann). Einer unserer lokalen Partner im Chintang Teilprojekt war Lokendra Tele, ein Grundschullehrer, der selbst schon vor unserer Ankunft damit begonnen hatte, Ritualtexte und Geschichten zu sammeln, d.h. aufzuschreiben. Er hatte schon einmal einen Kurs von Feldlinguisten des „Summer Institute of Linguistics“ (SIL) besucht und wusste deshalb, wie man Devanagari für die Transkription adaptieren kann. Wir gaben hierbei noch diverse Ratschläge und unterstützten ihn dabei, die Texte in einem kleinen Buch zu veröffentlichen. Daraus resultierte schließlich das Werk „Chintang Sanskriti ra Jalpa Devi“ (Chintang Kultur und Jalpa Devi), Kathmandu 2006. Dies kann als eine Art indigener Ethnographie gesehen werden, zumal der Autor eine Einführung in die verschiedenen kulturellen Besonderheiten der Chintang gibt. Der Band enthält aber vor allem ein umfassendes Korpus von Ritualtexten mit Nepali-Übersetzung.

Wie ging der Autor hierbei vor? Die Methode von Lokendra Tele kann als ein „skripturaler“ Ansatz beschrieben werden. Er ging davon aus, dass es nur einen richtigen, authentischen Text gibt, den er von seinen verschiedenen handschriftlichen Aufzeichnungen von performierten Rezitationen zu (re-) konstruieren versuchte. Dies ist nicht weiter überraschend bei einem Schullehrer. Doch die Auffassung, dass es einen richtigen Text gibt, ist sicherlich auch durch die Begegnungen mit der „Großen Tradition“ der Hindus geprägt, die in Ostnepal durch die Immigration von Hochkastigen verbreitet wurde. In Chintang gibt es, so sei hier angemerkt, sogar einige Haushalte von Kabirpanthis: Rai, die vor über hundert Jahren ihre tribale Religion abgelegt haben und zu diesem Bhakti-Orden konvertiert sind.

Für Lokendra machte die Aufzeichnung von verschiedenen Versionen desselben Rituals wenig Sinn. Dazu kam, dass er die Vermischung mit Nepali-Termini, die heute einen wichtigen Teil der Chintang Ritualsprache ausmachen, zu eliminieren versuchte: er ersetzte in seinen eigenen Texten diese Termini so 
gut er konnte und „korrigierte“ damit die vermeintlich korrupten Versionen. Lokendra versuchte also, ein kulturelles Ideal darzustellen, das es in der Wirklichkeit nicht mehr gab (und vielleicht auch so nie gegeben hatte). Er konstruierte also eine gereinigte Chintang Tradition. Die praktizierenden Ritualisten interessierte dies bislang jedoch kaum. Sie sehen die Anrufungen nach wie vor als etwas, das keiner Schrift bedarf.

Im Falle der Puma Rai sind wiederum andere Prozesse zu beobachten. Dort gibt es vor allem in der jüngeren Generation (Studenten, Arbeitsmigranten in der Diaspora) viele, die sich für die Erhaltung der eigenen Sprache einsetzen. Sie haben eine eigene Organisation gegründet („Puma Tupkha Bangkhala"), die sich dieser Aufgabe widmet, und einige Aktivisten bringen sogar eine Zeitschrift heraus („Bungwaphob“), die sich in der Puma-Sprache (in Devanagari) mit der eigenen Kultur befasst. Hier werden auch Puma-Gedichte, Puma-Kurzgeschichten und Puma-Lieder veröffentlicht, wie auch Artikel zu Geschichte, Ritualen und Festen. Diese Publikation ist allerdings eher an die Mittelschicht in den Städten gerichtet, es geht darin auch um Probleme des modernen Lebens (Computer, Film). Ritualtexte spielen hier keine gesonderte Rolle. Diese Puma-Aktivisten waren aber stark an unseren Filmaufzeichnungen von Ritualen interessiert. Gegenwärtig sind wir dabei, ein „Community Portal“ im Web aufzubauen, so dass die Archiv-Daten auch mit einer Puma-Oberfläche zugänglich sind. Für die Jüngeren, so scheint es, ist die Schrift nicht mehr der Maßstab aller Dinge. Sie sehen inzwischen die mündliche Tradition nicht mehr als per se minderwertig und nutzen moderne Medien (CD, DVD, Internet), um sie zu erhalten, zu repräsentieren und damit zu objektivieren. ${ }^{13}$ So kann der schriftliche Text auch ersetzt werden durch einen „Multimedia-Text“, d.h. einen mit Video und Audio synchron verknüpften Text, wie dies in unserem Archiv zu finden sein wird. ${ }^{14}$

\section{Schluss}

Die Situation heute ist also vielfältig und im Fluss: es ist noch nicht abzusehen, wie sich die oralen Traditionen in den nächsten Jahrzehnten entwickeln. Einige sind stark bedroht und werden verschwinden, andere werden sich als schriftliche Tradition erhalten, dabei aber einen grundlegenden Wandel durchlaufen, und es wird auch sicher Traditionen geben, die sich trotz allem weitgehend unverändert fortsetzen werden. Doch wie auch immer diese Prozesse verlaufen werden, die Positionierung gegenüber der Schriftlichkeit wird dabei eine wichtige Rolle spielen. Neben einer zunehmenden Verschriftlichung (in verschiedenen Schriften), wird es auch zu einer zunehmenden Literarisierung (in Sinne Pollocks) kommen: Selbst kleine Gruppen wie die Puma sind dabei, eine eigene Literatur auszubilden, wobei diese nicht mehr nur religiösen Charakter hat.

Gleichzeitig aber wird die Schrift ihre Vormachtstellung als Medium einer „textuellen Kohärenz“ gegenüber der vorgängigen „rituellen Kohärenz“ (s. Assmann 1997) tendenziell einbüßen, denn die neuen Medien erlauben eine neue Form des kulturellen Gedächtnisses. Ob dies jedoch zu einer Reoralisierung der Kultur führt (wie dies Marshal McLuhan vor langer Zeit prognostiziert hat) und eine spezifische „moderne Oralität““ (Schröder/Voell 2002) hervorbringen wird, bleibt abzuwarten. Möglich ist dies, doch wird dies sicherlich keine Rückkehr zu früheren Verhältnissen sein. Vielmehr wird sich das Selbstverständnis der Tradition, mithin die religiöse Identität, weiterhin grundlegend verändern.

Martin Gaenszle ist Professor am Institut für Südasien-, Tibet- und Buddhismuskunde, Universität Wien.

\footnotetext{
${ }^{13}$ Frühe Formen der Alphabetisierung in Nepal (zu Beginn des 20. Jahrhunderts) wurden von Laura Ahearn untersucht (2004).

${ }^{14}$ Siehe auch Liechty 2003, eine Ethnographie der neueren Formen von Medienkonsum in Nepal.
} 


\section{Literatur}

Ahearn, Laura. 2004. Invitations to love: literacy, love letters, and social change in Nepal. New Delhi: Adarsh Books.

Anderson, Benedict. 1983. Imagined communities: reflections on the origins and spread of nationalism. London: Verso.

Assmann, Jan. 1997. Das kulturelle Gedächtnis: Schrift, Erinnerung und politische Identität in frühen Hochkulturen. München: C.H. Beck (2. ed.).

Basso, Keith. 1974. The ethnography of writing, in: Bauman R. and J. Sherzer (ed.), Explorations in the ethnography of speaking. Cambridge: Cambridge University Press, 425-32.

Blackburn, Stuart. 2001. The tale of the book: storytelling and print nineteenth-century Tamil, in: Dwyer, R. and C. Pinney (ed.), Pleasure and the nation: the history, politics and consumption of public culture in India. New Delhi: Oxford University Press, 115-138.

Caplan, Lionel. 1970. Land and social change in East Nepal A Study of Hindu tribal relations. London: Routledge and Kegan Paul.

Chalmers, Rhoderick. 2003. Pandits and pulp fiction: Popular publishing and the birth of Nepali print capitalism in Banaras. Studies in Nepali History and Society 7/1: 35-97.

Chemjong. Iman Singh. 1961. Kirat Mundhum (Kirat ko veda) Dharan: Harkajang Ijam (2 ${ }^{\text {nd }}$ edition).

Crystal, David. 2000. Language death. Cambridge: Cambridge University Press.

Dhungel, Ramesh. 2006 (B.S. 2063). Sirijaṅgā hān ra limbu jātīya punarūtthānmā dhārmik-sāṃskṛtik prabhutvavādko praśna. Bahāḥ-Jarnal 3/3: 1-12.

Ekvall, Robert. 1964. Religious observances in Tibet: patterns and function. Chicago: Chicago University Press.

Gaenszle, Martin. 2000. Origins and migrations: kinship, mythology and ethnic identity among the Mewahang Rai of East Nepal. Kathmandu: Mandala Book Point \& The Mountain Institute.

Gaenszle, Martin. 2002a. Ancestral voices: oral ritual texts and their social contexts among the Mewahang Rai in east Nepal. Münster, Hamburg, London: LIT Verlag.

Gaenszle, Martin. 2002b. Countering the great traditions: Remakings of the Kiranti past, in: HarneitSievers, A. (ed.), A place in the world: New local historiographies fram Africa and South Asia. Leiden, Boston, Köln: Brill, 331-346.

Gaenszle, Martin, Balthasar Bickel, Goma Banjade, Elena Lieven, Netra Paudyal, Arjun Rai, Ichchha Rai, Manoj Rai, Novel Kishor Rai, Vishnu Rai, Narayan P. Sharma, and Sabine Stoll. 2005. The Chintang and Puma Documentation Project (CPDP). European Bulletin of Himalayan Research 28: 90-97.

Gaenszle, Martin. 2007. Schriftund Identität:Phalgunandaund die Anfänge der Kiranti-Sprachbewegung, in: Pfaff-Czarnecka, J. and C. Büschges (ed.), Ethnisierung des Politischen. Identitätspolitiken in Lateinamerika, Asien und den USA. Frankfurt: Campus, 284-307.

Hardman, Charlotte E. 2000. Other worlds: notions of self and emotion among the Lohorung Rai. Oxford, New York: Berg.

Höfer, András. 1981. Tamang ritual texts I. Preliminary studies in the folk religion of an ethnic minority in Nepal. Wiesbaden: Franz Steiner Verlag.

Kainla, Bairagi. 1994 (B.S. 2051). Sāmsoghā mundhum: pretātmako ākhyān ra anușthān. Kathmandu: Limbu Sahitya ra Sanskriti Utthan tatha Prakash Samaj. 
Kainla, Bairagi. 1996. Tongsing Mundhum of the Limbu ethnic group in the Kirat Mundhum. Journal of Nepalese Studies 1/1: 33-62.

Liechty, Mark. 2003. Suitably modern: Making middle-class culture in a new consumer society. Princeton: Princeton University Press.

Oppitz, Michael. 2006. Die Geschichte der verlorenen Schrift. Paideuma 52: 27-50.

Pollock, Sheldon. 1998. India in the vernacular millenium: literary culture and polity, 1000-1500. Daedalus 127/3: 41-74.

Schieffelin, Edward. 1998. Problematizing performance, in: Hughes-Freeland, F. (ed.), Ritual, performance, media. London: Routledge, 194-207.

Schlemmer, Grégoire. 2004. Inventing a past for inheriting a future: New visions of history among the Kirant intellectuals of Nepal. European Bulletin of Himalayan Research 25/26, Special Double Issue: Representing Local Histories in the Himalayas: 119-144.

Schröder, Ingo W., and S. Voell. Editors. 2002. Moderne Oralität: ethnologische Perspektiven auf die plurimediale Gegenwart. Marburg: Reihe Curupira.

Subba, Chaitanya. 1995. The culture and religion of Limbus. Kathmandu: K.B. Subba.

Waterhouse, David (ed.). 2004. The origins of Himalayan studies: Brian Houghton Hodgson in Nepal and Darjeeling 1820-1858. London and New York: Routledge Curzon. 